\title{
SIGNIFICADOS DEL OCIO Y LA CALIDAD DE VIDA EN PROFESORES DE UNIVERSIDADES IBEROAMERICANAS
}

\author{
Selma Aparecida Leite de Andrade', Maria Lucia Rodrigues ${ }^{2}$, \\ Aurora Madariaga Ortuzar ${ }^{3}$, Malvina Cruz Rentería ${ }^{3}$, Rosely Cubo Almeida ${ }^{4}$ e Savio \\ Guimarães $^{5}$

\footnotetext{
'Universidade Federal de Goiás UFG Regional Goiás, Brazil. leite_selma@ufg.br

${ }^{2}$ Pontíficia Universidade Católica de São Paulo, Brasil. leite selma@ufg.br

${ }^{3}$ Universidade de Deusto, España. malvicruz@deusto.es

${ }^{4}$ Universidade de Aveiro. Portuga. roselycubo@gmail.com

${ }^{5}$ Centro Universitário de Brasília, Brazil. savio.guimaraes.2010@gmail.com
}

\begin{abstract}
Resumen. Este estudio, en fase inicial, considera que, debido a la histórica diferencia en los aspectos sociodemográficos, económicos y culturales, existen heterogeneidades significativas en el medio universitario iberoamericano entre docentes de universidades latinoamericanas y europeas. Partiendo de la concepción del ocio como parte del desarrollo humano, nuestro objetivo es identificar qué significado tiene el ocio en docentes activos de universidades iberoamericanas en relación con su contexto sociocultural, sistema de valores, incluyendo la actual preocupación por el medioambiente. Asimismo, en el marco del acceso a derechos básicos de salud y bienestar, contrastar la percepción de los docentes de universidades iberoamericanas respecto de su calidad de vida, de su posición ante la vida, sus expectativas, objetivos, preocupaciones. Nuestra investigación es de carácter mixto. A través del método cualitativo y el cuantitativo nos aproximamos a docentes de universidades de países iberoamericanos activos en el período de 2017 a 2019. Para el propósito de la investigación recurriremos a la entrevista cerrada. En cuanto a la percepción de la calidad de vida, recurriremos al cuestionario WHOQOL bref, de la OMS.
\end{abstract}

Palabras-clave: Educación; Enseñanza Superior; Docencia; Calidad de Vida; Ocio.

\section{MEANINGS OF LEISURE AND QUALITY OF LIFE IN PROFESSORS FROM IBERO AMERICAN UNIVERSITIES}

\begin{abstract}
This study, in initial phase, considers that due to the historical difference in sociodemographic, economic, and cultural aspects, there are significant heterogeneities in the Ibero-American university environment between professors from Latin American and European universities. Starting from the conception of leisure as part of human development, our objective is to identify the meaning of leisure in active professors of Ibero-American universities in relation to its sociocultural context, value system, including the current concern for the environment. Likewise, within the framework of access to basic rights of health and well-being, contrast the perception of the professors of Ibero-American universities regarding their quality of life, their position before life, their expectations, objectives, concerns. Our research is of a mixed nature. Through qualitative and quantitative methods, we approach professors from universities in Ibero-American countries which were active in the period from 2017 to 2019. For the purpose of the investigation, we will resort to the closed interview. Regarding the perception of quality of life, we will use the WHOQOL bref questionnaire from the World Health Organization.
\end{abstract}

Keywords: Education; Higher Education; Teaching; Quality of life; Idleness.

\section{INTRODUCCIÓN}

En este estudio, seguimos a la Organización Mundial de la Salud en la definición de calidad de vida. Esta es "la percepción del individuo sobre su posición en la vida en el contexto cultural y el sistema de valores en el que vive y con respecto a sus metas, expectativas, normas y preocupaciones" (World Leisure and Recreation Association, 1994, p. 25). 
Por otra parte, el ocio es definido como fenómeno social, experiencia integral y como derecho básico que subraya su contribución al desarrollo humano en sus vertientes personal, social, cultural, económica y medioambiental. En nuestra hipótesis, los docentes de universidades iberoamericanas perciben deficiencias en la calidad de vida, que comprometen su salud física, psicológica, sus relaciones sociales y ambientales. Consideran que existe poca atención institucional en cuanto a políticas que aseguren a los trabajadores de la educación el desarrollo personal, social, cultural, económico y medioambiental. Además, no se advierte la concepción del ocio como derecho y experiencia integral en su vida.

Centrándonos en sus percepciones, tanto de la calidad de vida como del interés y la importancia del ocio como parte del desarrollo humano, se observan ciertas diferencias en cuanto a sus preocupaciones de acuerdo con su propio contexto sociocultural. Potenciar las políticas públicas es una prioridad para asegurar así el ocio como derecho básico y, en consecuencia, la salud, el bienestar y la calidad de vida de los seres humanos pertenecientes al ámbito de la educación.

La cuestión principal de nuestra investigación es: cuál es la percepción de los significados de calidad de vida y ocio en los docentes activos de universidades iberoamericanas, y cómo se evidencia, en la práctica, en el acceso a derechos básicos: salud física, psicológica, relaciones sociales y ambientales.

Como objetivos específicos elegimos: a) Coordinar el intercambio entre la Pontificia Universidad Catolica São Paulo; Universidade Federal de Goias - Regional Goiás UFG, en Brasil, y la Universidad de Deusto, en Bilbao, País Vasco; b) Revisión documental y de la literatura del estado de la cuestión del tema en estudio; c) Reuniones del grupo de trabajo encargado del intercambio internacional y de las encuestas; d) Construir una heramienta de trabajo para recoger información acerca del tema de estudio; e) Adaptar el instrumento WHOQOL-bref de la Organización Mundial de la Salud OMS para avalar la calidad de vida de los docentes activos de las universidades iberoamericanas; f) Mapear y especificar las universidades en el espacio iberoamericano; g) Identificar docentes activos de las universidades iberoamericanas y establecer contacto con ellos y/o ellas para la aplicación de la encuesta. 
Las encuestas WHOQOL-bref y Ocio. Después de la apreciación, sugerencias y aprobación de los asesores, se aplicará el pretest a los docentes involucrados en el proceso de estudio para ajustar el contenido y tiempo de contestación. Después de los ajustes coordinados, se aplicará el instrumento WHOQOL-bref, de la Organización Mundial de la Salud OMS, para recoger impresiones acerca de la calidad de vida de los docentes activos de las universidades iberoamericanas. Se aplicará asimismo el instrumento utilizado por el Instituto de Estudios de Ocio, de la Universidad de Deusto, para tipificar las actividades de ocio percibidas como más significativas para los docentes de las universidades iberoamericanas.

Finalmente, se diagramará e interpretarán los datos recibidos a través de las encuestas. Se establecerán vínculos, afinidades y diferencias entre las percepciones del grupo de estudio acerca de calidad de vida y significado del ocio en los ámbitos de la salud física, psicológica, relaciones sociales y ambientales como experiencia integral. Análisis, contrastes y síntesis serán recogidos en un documento propuesto para su publicación en forma de artículo, presentando antes los avances en un Seminario Internacional.

Con una metodología basada en datos cualitativos y cuantitativos, creemos que esta investigación puede contribuir en la reflexión sobre las necesidades presentes en el campo académico universitario, concretamente en lo que se refiere al derecho a la calidad de vida y ocio de los docentes universitarios. De la misma manera, subrayar y promover las potencialidades en favor de toda la comunidad humana de la que la universidad como institución forma parte.

\section{METODOLOGÍA}

A través de un acercamiento mixto, métodos cualitativo y cuantitativo, el estudio se desarrollará en tres etapas: a) Exploración; b) Trabajo de Campo y c) Análisis y tratamiento del material empírico y documental.

\subsection{Exploración}

La revisión de literatura y documentos es transversal al proyecto. Este paso de la investigación cualitativa permite la apertura a diferentes perspectivas y reflexiones, en suma, comprender, interpretar y reflexionar sobre el tema de estudio (Minayo, 2012 apud Minayo \& Costa, 2019, p.9). Es importante tener en cuenta que la experiencia humana se hace a partir de individuos concretos en el contexto de una historia colectiva, comunitaria e institucional. 
La historia individual está envuelta en la cultura del grupo en que se inserta (Minayo \& Costa, 2019, p. 10). Es necesario señalar que la organización de la bibliografía sobre temática iberoamericana y documentos históricos e institucionales sobre la universidad, formación, enseñanza, Calidad de Vida y Ocio, es esencial en la interlocución con el objeto de estudio. Asimismo, el conocimiento de las técnicas y herramientas metodológicas cuantitativas y cualitativas como Word Health Organization Quality of Life (WHOQOL).

\subsection{Trabajo de Campo}

Para el trabajo cualitativo empírico elegimos la técnica de entrevista cerrada por correo electrónico - google forms-. Buscamos recoger información en relación con la realidad que vive el o la docente, sobre su propia situación y, en general, aquellas experiencias que, como afirman Minayo y Costa, expresan modos de pensar, sentir, actuar y proyectar el futuro por parte de una persona o de un grupo, cuya base es necesariamente sociocultural (Minayo \& Costa, 2019). Para el propósito de la investigación y abordaje del tema optamos por la entrevista cerrada. Para las preguntas sobre calidad de vida presentamos el cuestionario WHOQOL bref, de la Organización Mundial de la Salud. Este ha sido producido con perspectiva internacional y con la colaboración de 15 centros de diferentes países, logrando desarrollar y validar una medida por la que se puede conocer la calidad de vida en diferentes culturas y, de manera específica, determinar la calidad de vida desde lo que es importante para el desarrollo de los individuos. La versión aquí recogida es abreviada y contiene 26 ítems de la versión original, que consta de 100 cuestiones. La WHOQOL-BREF produce un perfil de 4 dimensiones: salud física, salud psicológica, relaciones sociales y ambientales. Después de la presentación del Consentimiento de Uso de Datos, la encuesta comienza con un breve perfil del docente, pasando, a continuación, a preguntas abiertas sobre Ocio.

El universo de investigación consiste en docentes de universidades de países iberoamericanos. Los criterios en la muestra son:

a) docentes que estuvieron activos entre los años 2017, 2018 o 2019;

b) forman parte del objeto de estudio: Brasil, España, Portugal y Perú;

c) otros países iberoamericanos más poblados - México, Argentina, Colombia;

d) países iberoamericanos menos poblados: Costa Rica o Puerto Rico, o Panamá, y El Salvador. 


\subsection{Análisis y tratamiento del material empírico y documental}

Siguiendo a Minayo \& Costa, (2019, p. 26-30), el trabajo de análisis tiene algunas etapas, a saber: ordenación y organización del material empírico o secundario y la interpretación. Los autores hablan de la categorización, es decir, la búsqueda de las unidades de análisis en los textos, las referencias, el material de observación, los documentos, las entrevistas. Por último, la interpretación de segundo orden, que corresponde a la comprensión propiciada por la lectura atenta, profundizada e impregnada.

\subsection{Herramienta:}

a) Consentimiento de Uso de Datos

b) Perfil

c) WHOQOL BREF - Este cuestionario tiene como objetivo conocer su opinión acerca de su calidad de vida, su salud y otras áreas de su vida.

d) Concepto de Ocio, entendido como fenómeno social, experiencia integral y como derecho básico que contribuye al desarrollo humano en sus vertientes personal, social, cultural, económica y medioambiental.

e) Apreciación - opcional.

F) Disponibilidad en internet (https://forms.gle/L2hbNuecW1CCXBiLA)

\section{ANÁLISIS}

Consideramos que los procedimientos expuestos en los parágrafos anteriores nos acercan a los objetivos propuestos en conjunto. Siendo el objetivo principal identificar cómo perciben los docentes activos de las universidades iberoamericanas los significados de calidad de vida y ocio, la parte correspondiente a la etapa aquí expuesta consiste en analizar la propuesta, aclarar el proceso y configurar mejor nuestro objeto en el campo investigado, teniendo a la base el importante concepto de ocio.

Centrándonos en las percepciones de los docentes, tanto de la calidad de vida como del interés y la importancia del ocio como parte del desarrollo humano, se observan ciertas diferencias en cuanto a sus preocupaciones enmarcadas en su propio contexto sociocultural. 
La comprensión del concepto del trabajo en relación con el ocio en las universidades es determinante para el desenvolvimiento de los docentes en sus respectivas universidades. La precariedad de las políticas en favor del ocio y la calidad de vida de los docentes precariza, además de su salud y bienestar, la investigación y la extensión en el ámbito educativo.

En vistas a la comprensión global del ocio como favorecedor de los procesos de calidad de vida, seguimos la vertiente humanista (Cuenca Cabeza, 2003), que resalta la importancia de la satisfacción vital y gratitud en pro de la equidad, pluralidad, diversidad, alteridad en las interrelaciones del sujeto consigo, con los demás y en los entornos eco-naturales.

El ocio es signo de expectativa de vida hasta intensos momentos/aquí-ahora (Moreno, 1987). Se trata de una experiencia íntima en la que la experiencia del tiempo y del espacio es diferente a la marcada por las agendas o el aprisionamiento tiránico de la cronología del reloj. Junto a la presencia aparece también el disfrute de pertenencia (Azevedo, 2020), de intensos vínculos de proximidad y convivencia (Buber, 1995).

En cuanto a una vivencia gustosa y gratificante, el ocio es innovación que suple, en gran parte, las necesidades que antes se satisfacían por medio del trabajo, pero ahora, bien por su escasez, bien por su carácter competitivo, resultan de una realización harto difícil (Cuenca Cabeza, 2018).

Combinadas ambas concepciones, desarrollo e innovación, el ocio cuyo fundamento es la afectividad (Merengué, 2001) se constituye como facilitador de la trilogía pensar-sentiractuar: en lo que somos y cómo (identidad), en el compromiso de apertura, respeto y comunicación con los demás, y en la conciencia respecto de los entornos ambientalecológicos.

Una de las razones de más peso a la hora de identificar el ocio como 'presenciapertenencia' en docentes de universidades iberoamericanas es examinar sus experiencias: fuente de bienestar subjetivo, de aprendizaje y de actualización de papeles/roles socioculturales. De la misma manera, evidenciar las capacidades que despliegan para elegir vivencias que expanden sus campos cuánticos de energías positivas (Braden, 2008), de acuerdo con sus propias necesidades, intereses y preferencias. 
Finalmente, en cuanto a la presencia, de qué manera los docentes optan por compartir conocimientos; y, en línea con la pertenencia, cómo conforman principios y valores manifiestos de libertad y solidaridad.

Así, ocio y calidad de vida no tienen relación con el estatus socioeconómico, sino con las experiencias que los docentes absorben de vivencias de ruptura de su cotidianeidad (Aguado et al., 2008: 203; Kleiber, Walker y Mannell, 2011).

Partiendo de este presupuesto, es importante evidenciar cómo cada docente se percibesiente a sí mismo en relación con lo que vive. Como constructo psicosocial, los sentidos de ocio en vías de calidad de vida hacen referencia a la evaluación que cada uno experimenta mediante la moderación del self/ego y la suspensión de los juicios. Más allá de las conceptuaciones, ocio y calidad de vida se vinculan estrechamente a través de las experiencias de creatividad-espontaneidad, experiencia lúdica y festividad-felicidad.

En síntesis, se trata de absolver cuestiones importantes de fondo: ¿Se puede comprobar que los profesores perciben experiencias positivas de ocio? ¿Cuáles vivencias les proporcionan satisfacciones emocionales, sociales, fisiológicas, conductuales y cognitivas que complementan su existencia y empoderan su valor como ciudadanos? A nivel de competencias y habilidades relacionadas con la salud y el funcionamiento físico y mental, ¿cómo se perciben en el proceso de envejecimiento?

A medida que avanzan las investigaciones (Alencar, 1997; Christóvão, 2004; Almeida, 2007; Mészaros, 2008; Cabrera, 2010; Andrade, Gomes, Knobel e Silva, 2012; Cubo, 2018; Cubo Pinto de Almeida, 2019), se confirma la existencia de una estrecha relación entre bienestar psicosocial, satisfacción vital, experiencias de ocio e innovación y desarrollo en proyectos de vida de profesionales de la enseñanza superior.

Dos documentos internacionales, especialmente reconocidos por los investigadores del ocio, como son la Carta Internacional de Ocio y Para la Educación del Ocio, de la World Leisure and Recreation Association (WL, 1994 y 1998), señalan como primeria medida que el ocio contribuye al ejercicio de una ciudadanía democrática para aumentar la calidad de vida.

\section{CONCLUSIÓN}

Como proyecto en construcción, necesitamos tiempo y trabajo para completarlo. 
Tenemos lista una herramienta que puede ser un material abundante para estudiar nuestro objeto y buscar conocer cuáles son las percepciones de los docentes de universidades iberoamericanas respecto de su calidad de vida, de su posición ante la vida, sus expectativas, objetivos, preocupaciones, y qué significado tiene el ocio en su contexto cultural y sistema de valores.

Ciertamente, creemos que cuanto más satisfecho esté el docente en su experiencia vital multidimensional en los diferentes contextos culturales, sociales y medioambientales, mayor será la comprensión del ocio como experiencia integral y derecho básico para el desarrollo humano. Creemos que nuestro interés en la investigación acerca de las percepciones de los docentes de universidades iberoamericanas, en lo que implica significados de calidad de vida y ocio, puede permitir una mayor y mejor reflexión sobre las muchas necesidades presentes en el ámbito académico universitario.

En esa medida, identificar las potencialidades que pueden desarrollarse a partir de un cambio de percepción en lo que corresponde a derechos básicos, dentro de los cuales, como hemos indicado, se encuentra el ocio. Finalmente, prestar más atención, como docentes, a los espacios en los que nos movemos y a los que poca reflexión prestamos por estar enfocados en investigaciones asociadas, más allá de los límites socioespaciales de las universidades, con la esfera social.

\section{REFERENCIAS}

Aguado, O.; Fernández, M.; Vaz, P.; León, J.C. (2008). Políticas públicas, calidad de vida e interculturalidad. Revista del Ministerio de Trabajo e Inmigración (75).

Alencar, O. (1997). O estímulo à criatividade no contexto universitário. SciELO Brasil.

Almeida, N. F. (2007). Universidade nova: textos críticos e esperançosos. Salvador: EDUFBA

Andrade, Gomes, Knobel e Silva (2012). Programa de formação interdisciplinar superior: um novo caminho para a educação superior. Revista Brasileira de Estudos Pedagógicos v. 93, n. 235, p. 698-719, set./dez.

Azevedo, C. (2020). O corpo como campo informacional. Fortaleza: Orion com Saber.

Braden, G. (2008). A matriz divina. São Paulo: Cultrix.

Buber, M. (1995). Eu e tu. São Paulo: Moraes.

Cabrera, J. (2010). Transdisciplinaridad en la formación universitaria:una investigación en curso. En Torre y Pujol (coord.) Creatividad e innovación. Enseñar e investigar con otra conciencia, p. 177-191. Madrid: Rd. Universitas S.A.

Christóvão, M. C. T. (2004). A formação permanente do educador e o processo ensino aprendizagem. Gestão Educacional: Uma nova visão. Cap. X, p. 171. Porto Alegre: Artmed. 
Cubo, R. (2018) Ócio e trabalho: dimensões compartilhadas. O sentido do ócio redefinindo tempos e espaços organizacionais. Revista Científica Multidisciplinar Gentil Alves Pessoa,v. $\begin{array}{llllllllll}1 & \text { n. } & 1\end{array}$ .http://www.revistaicgap.com.br/index.php/icgap/article/view/14

Cubo, R.P.A. (2019). Ocio como ámbito de integración de inmigrantes: representaciones y vivencias de mujeres brasileñas en el País Vasco. Revista Subjetividades, 19(2), e9229. https://periodicos.unifor.br/rmes/article/view/e9229

Cuenca Cabeza, M. (2003). Ocio humanista: dimensiones y manifestacion es actuales del ocio. Instituto de estudios de ocio. Bilbao: Universidad de Deusto.

Cuenca Cabeza, M. (2018). El papel del ocio enla transformación de las ciudades: reflexiones desde el caso Bilbao.(Orgs.) Cláudio Augusto Silva Gutierrez, Fernando Edi Chaves. Ócio e cidade: o papel do ócio na transformação das cidades.São Leopoldo: UNISINOS.

Kleiber, D.A; Walter, G.J; Mannell, R.C. (2011). A social psychology of leisure. Nova York: Venture Pub.

Merengué, D. (2001). Inventário de afetos: inquietações, teorias, psicodramas. São Paulo: Ágora.

Mészaros, I. (2008). A educação para além do capital. São Paulo: Boitempo.

Minayo, M. C. S., Costa, A.P. (2019). Técnicas que hacen uso de la palabra, de la observación y de la empatía. Aveiro, Ludomedia.

Moreno, J. L. (1987). Psicodrama. São Paulo: Cultrix.

WL (World Leisure and Recreation Association) (1994). International Charter for Leisure Education, enRevista ELRA (European Leisure and Recreation Association), summer, 13-16. Edición en español en Cuenca Cabeza, M. (2004) Pedagogía del ocio: modelos y propuestas, p. 315-324. Bilbao: Universidad de Deusto.

WL (World Leisure and Recreation Association) (1998). Carta internacional del ocio. Edición en español en Cuenca Cabneza, M. (2004): Pedagogía del ocio: modelos y propuestas, p. 325-327. Bilbao: Universidad de Deusto 